\title{
Periodización táctica y la toma de decisiones en la transición ofensiva en el baloncesto femenino de la ESPOCH sede Morona Santiago Ecuador
}

Tactical periodization and decision-making in the offensive transition in women's basketball at the ESPOCH headquarters Morona Santiago Ecuador

\author{
Danilo Ortiz Fernández. ${ }^{1}$, Alberto Alzola Tamayo. ${ }^{2}$, José Alfredo Sánchez Anilema. ${ }^{3}$ \\ \& Omar Hechavarria Jeorge. ${ }^{4}$
}

Recibido: 25-04-2020 / Revisado: 22-05-2020 / Aceptado: 23-06-2020 / Publicado: 03-07-2020

\begin{abstract}
.
DOI: https://doi.org/10.33262/cienciadigital.v4i3.1343

Summary. This article aims to provide a work tool, to basketball coaches that allow them to help their players, in the decision-making process from the teaching and learning process in the offensive transition of basketball, female of the Polytechnic Higher School of Chimborazo Extension Morona Santiago. bridge between the previous article and this is proposed a Scheme of exercises that allows them to have a working guide based on the tactical periodization Our work sample was composed of 15 players, having as a methodological objective the application of tactical periodization in training, touching a aspect, important within the basketball game as is the offensive transition, looking for an adequate level of play enhancing its application in our team based on tactical periodization adapting its principles and methodology to our environment, allowing a better performance in our transition.

Keywords: Tactical prioritization offensive transition decision-making model game.

\footnotetext{
${ }^{1}$ Escuela Superior Politécnica de Chimborazo, Ecuador. danilo.ortiz@espoch.edu.ec https://orcid.org/00000002-4777-1495

${ }^{2}$ Escuela Superior Politécnica de Chimborazo Sede Morona Santiago. Ecuador. alberto.alzola@espoch.edu.ec, https://orcid.org /0000-0001-9715-2091

${ }^{3}$ Escuela Superior Politécnica de Chimborazo. alfsan3@ hotmail.com

${ }^{4}$ Universidad Las Tunas, Cuba, omarhj5607@gmail.com
} 


\section{Resumen.}

El presente artículo tiene como objeto brindar una herramienta de trabajo, a los entrenadores de baloncesto que le permitan ayudar a sus jugadores, en la toma de decisiones desde el proceso de enseñanza y aprendizaje en la transición ofensiva del baloncesto, femenino de la Escuela Superior Politécnica de Chimborazo Extensión Morona Santiago se propone un Esquema de ejercicios que les permita tener una guía de trabajo con base a la periodización táctica Nuestra muestra de trabajo estuvo integrada por 15 jugadores, teniendo como objetivo metodológico la aplicación de la periodización táctica en el entrenamiento, tocando un aspecto, importante dentro del juego de baloncesto como lo es la transición ofensiva, buscando un adecuado nivel de juego potenciando su aplicación en nuestro equipo con base en la periodización táctica adecuando sus principios y metodología a nuestro entorno, permitiendo un mejor funcionamiento en nuestra transición ofensiva, desde el punto de vista individual (fortaleciendo la toma de decisiones) y con ello mejorar el preforman y el accionar como equipo, lo individual en función de lo colectivo.

Palabras claves: Priorización táctica transición ofensiva toma de decisiones modelo de juego.

\section{Introducción.}

En los últimos tres décadas, se han producido avances significativos en cuanto a los conocimientos en todos lo referente al ámbito deportivo, sistemas de entrenamientos, de enseñanza y metodologías. Señalándose que en los primeros años de la década del 90 se empieza a notar un mayor desarrollo en las formas de enseñanza, aunque, se siguen manejando estrategias y métodos de enseñanza de la pedagogía existentes. El baloncesto no es una excepción tendencia existente en la actualidad al tratar de mejorar los diferentes aspectos del juego tanto en el orden ofensivo y defensivo, desde lo individual y colectivo.

En opinión de Torrents (citado en Alzola et, al, 2019):

El entrenamiento deportivo ha estado influenciado por la tendencia mecanicista del ser humano. A pesar de que siempre se aluda necesidad de integrar todos los aspectos del entrenamiento y se propongan concepciones holísticas (integradas), la estructura sigue siendo una visión cartesiana que concibe a los organismos prácticamente como maquinas construidas por diferentes partes (p. 391)

Castro (2019), define desde una línea más contemporánea al aportar diferentes elementos que se tienen en cuenta en la actualidad sobre la base de investigaciones relacionadas con la preparación del deportista a través del tiempo “...es el proceso pedagógico de preparación del deportista, que tiene como fin el alcance de un optimó estado competitivo, desde el punto de vista físico, psicológico, técnico, volitivo, moral y táctico..." (p. 16). Elementos 
indispensables a la hora de enfrentar la planificación del entrenamiento deportivo en los diferentes deportes.

El baloncesto está enmarcado en un grupo de deportes denominados deportes de equipos o de colaboración/oposición, (Freyre, 2018, p.11), debemos decir que intervienen en su desarrollo diferentes factores estructurales que tienen que entrenarse de forma global e interrelacionados. Ello solo es posible mediante el entrenamiento integrado de todos estos factores mediante el juego, célula de la periodización táctica, metodología que se asume en el entrenamiento del equipo femenino de la ESPOCH.

En este sentido, asume la definición de periodización táctica aportada por Frade, (citado en Castro 2019), creador de esta teoría, la cual matiné su vigencia ya que es sustentada por los estudios de investigadores hasta la actualidad, definida como. “...una metodología de entrenamiento cuya preocupación máxima es el que un equipo pretende producir en la competición. Es por ello que el modelo de juego y la dimensión táctica se asumen como guías de todo el proceso de entrenamiento..." (p. 23)

Según Valero (2016) el Baloncesto es un deporte de equipo, un juego deportivo que se caracteriza por ser muy dinámico y de gran creatividad, los sujetos que lo practican están expuestos a diferentes situaciones que se presentan en el terreno y deben ser solucionadas. En este deporte el resultado depende del trabajo colectivo del equipo para lograr los objetivos (p. 22)

El juego de transición ofensiva se utiliza en un alto porcentaje de veces después de recibir canasta del equipo adversario. Son sistemas rápidos donde participan los 5 jugadores en cancha, aprovechan el despliegue a la zona de ataque y permite actuar con rapidez, antes que la defensa se organice, con el objetivo de sorprender y obtener ventajas sobre el adversario.

A diferencia del contraataque en que las decisiones de juego vienen determinadas por la defensa es decir el número de defensores (ventaja numérica 1x0, 2x1, 3x2 3x3 etc.) o por los espacios libres (ventaja por espacios libres $2 \times 2,3 \times 3$ ) en la transición la pauta de juego corresponde, al menos inicialmente al ataque.

El paso defensa- ataque de forma dinámica tiene 5 partes diferenciadas.

1. Posesión del balón: Puesta en juego del mecanismo de ataque desde el rebote o después de canasta. Organizar quien saca el balón desde el fondo de la cancha y como lo recibe el base. Velocidad en el primer pase de contraataque. los jugadores deben ser capaces de conseguir el balón y ponerlo en juego rápidamente.

2. Contraataque primario: Ocupación de calles, aperturas diversas con tres jugadores, llevar el balón por el centro traspasar rápidamente la línea de medio campo.

3. Contraataque secundario: Adaptación al ataque de los jugadores 4 y 5. 
4. Juego organizado: con variación de opciones.

5. Rebote ofensivo.

Todos estos elementos son esenciales para el desarrollo exitoso del juego, la base para que los entrenadores desarrollen estrategias "incidentales e intencionales" Raab, (citado en Suárez, Courel y Cárdenas, 2017)

Las primeras pretenden generar un aprendizaje auto-dirigido y basado en la práctica sin guiar, en la interacción del deportista con su entorno. Sin embargo, las segundas pretenden conseguir un aprendizaje dirigido por el entrenador que se encarga de guiarlo haciéndole consciente de los objetivos específicos de la práctica, así como de las claves atencionales que le ayudarán a una interpretación adecuada del con- texto de juego (p. 3)

Son elementos que contribuyen al buen funcionamiento del juego que los entrenadores tienen que ser capaces de que sus jugadores se apropien de ellos para una mejor toma de decisiones en las diferentes circunstancias del partido de Baloncesto, lo cual queda bien explícito en palabras de Cárdenas y Alarcón, (citado en Suárez, Courel y Cárdenas)

El aprendizaje de estas claves resulta determinante para reducir el nivel de incertidumbre al que está sometido el jugador, para predecir las acciones de los adversarios y, en consecuencia, para establecer conductas de anticipación (p. 3), en este sentido los entrenadores tienen que estar preparados para el uso de estas claves en situaciones cambiantes del juego

\section{Marco Teórico.}

En el baloncesto actual el dominio técnico por sí solo no constituye una garantía en el desarrollo del juego es por ello que en el proceso de enseñanza y aprendizaje, la conjugación de los elementos técnicos, táctico, psicológicos, a la hora de la enseñanza, tiene que ser una herramienta básica que todo entrenador tiene que poseer, para así formar jugadores con conceptos formativos de estos tres elemento del juego, permitiendo posteriormente contar con jugadores versátiles con amplios conocimiento de la lectura del juego en las distintas situaciones que se puedan presentar, partiendo de estos elementos ir formando estilos y modelo de juego.

El modelo de juego no se produce de forma natural ni aparece de la nada. El modelo de juego es el núcleo central, sobre la cual se trabajan en los entrenamientos. Este modelo de juego es planteado por el entrenador y es el resultado de un fenómeno construido a partir de diferentes factores, que determinan la forma de jugar de un equipo Tamarit (2007) para Fleitas es el modelo de juego será más rico cuando más posibilite a los jugadores ampliar su propia creatividad y talento en el juego. (citados en Alzola, et, al, 2019, p. 393)

En este particular la priorización táctica Frade, (citado en Castro 2019), argumentan una serie de elementos como especificidad que resaltan la importancia de entrenar jugando que en el 
contexto del entrenamiento del baloncesto y en particular la toma de decisión en la transición ofensiva:

Conseguir un Modelo de Juego lleva tiempo, por ello es necesario entrenarlo desde el primer día de entrenamiento y subordinarlo todo a la supradimensión táctica. Será el principio de Especificidad quien dirija la Periodización Táctica. La forma de jugar caracterizada por ciertos Principios y Subprincipios que conforman un Modelo de Juego (permitiendo la aparición del resto de dimensiones por arrastre) provoca que cada ejercicio esté dotado de una Especificidad del juego que queremos. O sea, siempre estamos entrenando nuestro juego. En esta Metodología, el Principio de Especificidad, que debe ser cumplido en todo momento del entrenamiento, pasa a determinarse como el Principio de Principios de la Periodización Táctica. (p. 24)

Es importan destacar que el modelo de juego no es algo empírico se construye en base a las experiencias de los entrenadores basadas, estudios propios y de otros, el poder trabajar con diferentes equipos, el querer busca a toda costa su propio modelo de jugo, es además pode contar con jugadores capaces, con un equipo de trabajo, que te apoye con fe en tus metas y objetivos, es historia cultura de aquellos lugares por donde trabajamos.

\section{Acercamiento a la toma de decisiones.}

Aspecto importante en el éxito del juego ya que se sustenta en la experiencia y preparación de preparadores y jugadores aspectos abordados por diferentes investigadores tales como: Gilovich, 1984; Bar-Eli, Plessner y Raab, 2011 y Richards, Collins y Mascarenhas, 2017 (citados en Suárez y Cárdenas 2017, p. 44) refieren al respecto:

- El deporte reúne características únicas que lo convierten en un laboratorio ideal para el estudio de la toma de decisiones, pues en él se involucran tanto la cognición como la acción

- Desde la planificación de entrenamientos hasta las decisiones in situ en competición se ha de buscar la máxima optimización, aumentando así la probabilidad de éxito final.

- Los juicios y la toma de decisiones juegan un papel fundamental en el éxito deportivo. Jugadores y entrenadores toman constantemente decisiones que repercuten de forma directa sobre los resultados obtenidos.

Bar-Eli, Plessner, \& Raab, (citados en Práxedes 2018), aportan una definición que a consideración de los autores del artículo define de manera sintética y bien próxima a la realidad en que se realiza la toma de decisiones en el juego de Baloncesto la consideran como: “...el proceso a través del cual los deportistas seleccionan una respuesta entre varias alternativas con el fin de ejecutarla en un momento determinado y en una situación real de juego..." (p. 54) 
Por su parte Stratton et al. 2004, (citado por Klimenko, Darío, o Múnera y Rave 2020) aporta elementos necesarios a tener en cuenta desde el punto de vista del jugador en la toma de decisiones:

Un jugador de un deporte de conjunto experto y exitoso necesita procesar una gran cantidad de información, en poco tiempo, además, bajo presión. Muchas decisiones deben tomarse rápidamente y reevaluarse dependiendo de la demanda en el campo. Uno de los elementos del juego exitoso es la toma de decisiones rápida y hábil, donde tanto la precisión como la velocidad están al máximo nivel. Tal comportamiento ayuda al jugador a "leer el juego", predecir el comportamiento de los oponentes y escoger jugadas acertadas. (p. 30)

La toma de decisión se entiende como una relación funcional entre el individuo y el ambiente. es un proceso complejo que no puede separarse de la expresión conductual del individuo.

Los actuales modelos ecológicos del sistema dinámico tienen su origen en la teoría de la percepción directa de Gibson, analizado por Araujo (citado en Suarez 2016) quien propuso que: “...las personas perciben y actúan sobre propiedades funcionales de las sustancias, superficies, lugares, objetos y eventos en el entorno. Estas propiedades proporcionan oportunidades de acción (affordances) y se definen por la acción de complementariedad entre las restricciones del ambiente (constraints)..." (p. 36)

Aspectos importantes sobre todo en las condiciones que se realiza el juego de Baloncesto (ambiento social), aficionados, jueces, la televisión y do equipos que pugnan por la victoria, que influyen en la toma de decisiones de los jugadores.

Lo anterior se ve reflejado en palabras de los investigadores, Davids, Güllich, Shuttleworth, \& Araújo, (2017), que sustentan: “...cuanto mayor y más diverso sea el rango de posibilidades de acción, más preparado estará el jugador para recibir información, adaptar sus acciones, tomar decisiones e interactuar más eficazmente con los constreñimientos impuestos por el entorno..." (p. 56)

Gutiérrez et al., (citado en Práxedes 2018), refiere la importancia que para la consolidación de habilidades y destrezas deportiva tienen la toma de decisiones, con las cuales se coincide: “...no son únicamente determinantes factores como la ejecución técnica de las habilidades, sino también aquellos relacionados con la toma de decisiones..." (p. 231)

La toma de decisiones de acuerdo con Bunker y Thorpe (citados en Cuesta, 2016) tienen dos elementos fundamentales en el accionar deportivo sustentadas en el: "qué hacer y cómo hacerlo" (p. 28)

En relación con la necesidad de promover la conciencia táctica en el primer aspecto y en caso de segundo, centrado más con la valides y calidad de la solución. Aspectos importantes para 
planificar ejercicios tácticos- decisionales que traigan consigo el logro de la transición ofensiva en el entrenamiento y el juego real. Aspectos importantes para un deporte como el Baloncesto de carácter abierto caracterizado por los ambientes estables que dependen de la eficiencia, eficacia y velocidad para la realización de las acciones del juego.

\section{La concentración y toma de decisión en los entrenamientos.}

Es preciso tener conocimiento para poder comprender e interpretar estos entornos situacionales, se precisa estar atentos, concentrados para poder tomar las decisiones correctas en el momento y el espacio precisos. En este sentido, mientras más concentrados estén los jugadores, aumentara en un gran porciento el poder tomar buenas decisiones y con ello el aumento del nivel de juego.

En este sentido Frade, citado en Castro (2019) enfatiza que: “...los buenos desempeños de entrenamiento reclaman elevada concentración para aquello que el entrenador pretende..." (p. 24), se está en consonancia con lo expresado por este autor ya la toma de decisiones requiere del máximo de atención de los jugadores involucrados en el juego para que este sea exitoso.

Lo anterior queda claro en las propias palabras de Frade cuando argumenta:

La Periodización Táctica pone énfasis en la asimilación de una forma de jugar, en sus principios: organización de la defensa, del ataque y de los principios que son sus fronteras, o sea el modo como se transita de un momento al otro, siendo que esto sólo se hace en concentración. (Frade, citado en Castro, 2019, p. 24)

El Baloncesto es un deporte rápido dinámico de desarrollo caótico, impredecible en cuanto a su accionar es por esto que el poder contar con una metodología como la periodización táctica, que nos permite trabajar de forma específica todo el accionar del juego.

De acuerdo con Castro (2019) la concentración especifica en la periodización táctica tiene que estar sustentada en ejercicios tácticos que logren la transformación del accionar del jugador a partir de ser portador de un conocimiento procedimental que le facilitan la toma de decisiones en el juego. (p. 25)

De ahí que se coincida con Serra (2013), en cuanto a la importancia de que los jugadores se apropien no solo de la técnica sino también de las herramientas tácticas que solo en el entrenamiento diario del juego se pueden adquirir:

"....ser un dechado de técnica no es suficiente para vencer en una situación determinada, es necesario tomar decisiones y desarrollar habilidades ajustadas a las limitaciones de cada situación de juego y contexto, y acordes a su vez a las representaciones mentales que se hacen en el juego de acuerdo al problema a resolver..." (p. 48) 
En este sentido, el entrenador debe jugar un papel activo contribuir en la comprensión de la nueva metodología ser un gestor de la misma, ser capaz de aglutinar sus deportista y colectivo técnico alrededor de esta nueva manera de entrenar. Partiendo de tres aspectos que a nuestro modo de ver son importantes.

- Aprender Jugando.

- Entrenar jugando

- Desarrollo Biopsicosocial de nuestros jugadores

Consideramos un, desarrollo físico atreves del juego filosofía de la priorización táctica

Desarrollo psicológico basado en el entrenar nuestro modelo de juego en situaciones de competición, proponiendo distintas variantes de complejidad, imponiendo a nuestros deportistas estar alertas siempre, para poder dar respuestas ante distintas situaciones de variabilidad del juego.

Desarrollo social dado por la constante interrelación entre jugadores al tener que jugar aprendiendo, entrenar jugando (priorizando el trabajo colectivo desde lo individual)

Aspectos determinantes en el éxito de las acciones del juego de Baloncesto, que junto a la preparación que tengan los entrenadores para el empleó de claves teniendo en cuenta las diferentes situaciones del juego. Ello implica el nivel técnico táctico de los jugadores y el nivel de complejidad de las interacciones que se producen en el juego entre compañeros y adversarios en la toma de decisiones para la transición ofensiva.

Lo cual ocurre en cuestiones de segundos decisivos en el juego real, permite al equipo acercarse en el marcador, empatar el juego, tomar la ventaja o ampliarla, de ahí la importancia del empleo por parte de los preparadores de estrategias de enseñanzas intencionales.

\section{Aproximación a la periodización táctica.}

La periodización táctica sustentada en el entrenamiento del modelo de juego deseado, que responda en todo momento a saber sobre ese saber hacer, es decir que los jugadores tengan una mentalidad que le permita sea cual sea el contexto encontrar la respuesta acertada a la problemática presentada.

En este sentido, Loaiza (2017) refiere que:

La periodización táctica es una forma de organización del entrenamiento de reciente aplicación en muchos deportes colectivos. Tamarit, (citado en Loaiza. Loaiza) este planteamiento genuino de Vítor Frade data de finales de los años 70, pero su implantación como estructura válida no se dio hasta la consecución por el FC Oporto de José Mourinho de numerosos éxitos gracias, en parte, a esta estructuración del entrenamiento (p. 3) 
Argumentando Tamarit, (citado en Loaiza 2017): Su creación no viene determinada de pensamientos teóricos surgidos de otras áreas, sino de tener el juego como eje central. Para ello, en primer lugar, hay que conocer profundamente la modalidad deportiva, para posteriormente aplicar los principios y conceptos del entrenamiento (p. 53).

Y puntualiza: La periodización táctica determina que el entrenamiento vendrá estructurado por el desgaste emocional y que éste dependerá de los siguientes factores Tamarit, (citado en Loaiza 2017, p. 53)

Otro aspecto a tener en cuenta a la hora de plantear la progresión de los ejercicios de acuerdo con Tamarit (2007) Complejidad de los principios que se trabajan: complejidad de la dinámica (reglas y objetivos del ejercicio), cantidad de jugadores (variable decisional), espacio de juego (velocidad de decisión del jugador) y tiempo de duración de ejercicios (tipo de ejercicio y principio a entrenar) (p. 54)

Es importante al planificar los ejercicios que estos estén en función de despertar en el jugador su creatividad, que lo reten a la solución de ejercicios tácticos complejos.

Otros conceptos que hay que destacar y distanciar de los métodos tradicionales son la intensidad y el volumen. La intensidad se refiere principalmente a la concentración, no a la velocidad de desplazamiento. Esto se debe a que los jugadores deben pensar y esto exige que estén concentrados. En cuanto al volumen, la periodización táctica lo entiende como un "volumen de intensidades" Loaiza (2017) (volumen generado a partir de repeticiones sistemáticas de principios y subprincipios) (Tamarit, 2007, p. 33).

En este sentido, Loaiza (2017), platea este método de entrenamiento totalmente específico sin entrenamiento en gimnasios o ejercicios descontextualizados, hace que sea necesario engañar al deportista para que se sienta cómoda con una estrategia de entrenamiento a la que no están acostumbrados, por lo hábitos de temporizaciones más convencionales. (p.53).

Por lo que se considera importante el planificar los ejercicios, en función de despertar en el jugador su creatividad, que lo reten a la solución de ejercicios tácticos complejos. Entrena en circunstancias de juego real diariamente, que la carga principal sea el trabajo táctico y todo lo demás se derive a partir de esto. que el atleta se construya secuencias de imagen a semejanza de lo que será el juego real y con ello adquirir conocimiento a través de la práctica diaria. Permitiendo una mejor comprensión del juego y así poder hacer los ajustes necesarios, y tomar las decisiones más acertadas en el menor tiempo posible en cada situación del juego.

\section{Contraataque y Transición ofensiva.}

El concepto de contraataque en baloncesto descrito por Roman (citado en Leica 2018) refiere: “...se basa fundamentalmente en la respuesta ofensiva veloz frente al avance del rival. Es decir, responder lo más rápido posible, con acciones efectivas, las acciones defensivas del 
equipo oponente. Estos dos conceptos: velocidad y efectividad son elementos clave en la ejecución del contraataque..." (p. 30)

Ataques intensos con altos requerimientos de intensidad y concentración mental deben estar ligados a defensas intensas y de alta concentración. Roman, (citado en Leica 2018 p. 31), lo planteado por este autor se entiende decisivo para la planificación de situaciones tácticas que impliquen estos dos elementos (ataque y defensa) que exigirán de los jugadores tomar decisiones apropiadas.

Entendemos la transición como describe Roman (citado en Leica 2018)

"...la fase de juego desde que termina el contraataque hasta que comienza el ataque posicional. Una transición es tanto más efectiva cuanto más orden y continuidad encontramos en su desarrollo. Debemos evitar, por tanto, diferencias de ritmo entre estas fases, provocando peligro en todas las acciones que se desarrollen. Debemos entender la transición como un continuo y no una fase intermedia de colocación de jugadores para desarrollar un ataque más estructurado (p. 31)

Estos argumentos son relevantes para la consecución de forma rápida y efectiva de las acciones del juego, lo cual permitirá sacar ventaja del contrario en el juego.

Por su parte, Carbelleira, (citado en Affonso y García.2017) denomina la transición como: "...la fase intermedia comprendida entre un contraataque que no ha finalizado en canasta y el inicio del ataque posicional. También plantea que estas fases dinámicas son altamente eficaces para conseguir el objetivo del ataque y generar situaciones ventajosas. A su vez nos explica que tanto el contrataque como la transición se basan en no dejar que la defensa se coloque..." (p. 6)

Así mismo, Calvo (Citado en Affonso y García.2017), plantea que: “...la transición ofensiva es un ataque organizado que parte de un contraataque, y sirve para unirlo con el ataque estacionado. Se utiliza cuando no se pudo obtener una canasta rápida en el contraataque..." (p. 14)

De igual manera Refoyo (citado en Affonso y García.2017), plantea que:

“...la transición debe tomarse como una continuidad dentro del juego y no como una fase intermedia de espera para que los jugadores tomen sus posiciones. Define la transición como la fase del juego comprendida entre el contraataque que no termina en canasta y el ataque posicional. Asimismo, menciona que no deben existir diferencias de velocidad entre una y otra fase para así tener la chance de provocar peligro en todas las acciones (p. 14)

Es importante destacar, que los autores antes misionados ponderan ambos factores (defensa y transición de juego) con lo cual se coincide, ya que tienen una relación importante. Ya que, dependiendo del alto grado de componentes, de intensidad, agresividad, concentración, ritmo 
y efectividad en defensa del equipo, se traduciría en un contrataque y transición ofensiva eficaz y contundente. Ello siempre y cuando los jugadores sean capaces de transferir estos componentes, la transición no es un elemento aparte es un eslabón de toda la cadena. Que conforma el juego de baloncesto con una visión holística, un elemento de conexión entre el contraataque y el juego posicional.

Consideramos que la base de una buena transición ofensiva, se sustenta fundamentalmente en el poder contar con una defensa eficaz en todos los aspectos de juego, contraataque estructurado y solo entonces la transición ofensiva podrá ser, veloz, segura con un buen por ciento de efectividad.

\section{Análisis de resultado.}

Al evaluar los datos arrojado por nuestras observaciones al instrumento de medición de nuestra muestra observamos un mayor por ciento en las categorías siempre y casi siempre, por lo que podemos concluir una influencia positiva de los ejercicios en nuestros estudiantes.

Tabla 1. Desarrollo y logro de los ejercicios

(S): SIEMPRE (CS): CASI SIEMPRE (PV): POCAS VESES (N): NUNCA

\begin{tabular}{lcccccccc}
\hline \multicolumn{1}{c}{ Categorías } & $\mathbf{S}$ & $\mathbf{\%}$ & $\mathbf{C S}$ & $\mathbf{\%}$ & $\mathbf{P V}$ & $\mathbf{\%}$ & $\mathbf{N}$ & $\mathbf{\%}$ \\
\hline Ocupación de carriles & 9 & 60,00 & 33,33 & 20,00 & 1 & 6.66 & - & - \\
$\begin{array}{l}\text { Superioridad numérica } \\
\text { Toma de decisión en la }\end{array}$ & 6 & 40,00 & 4 & 26,66 & 3 & 20.00 & 2 & 13,33 \\
$\begin{array}{l}\text { transición } \\
\text { Continuidad de juego }\end{array}$ & 8 & 53,33 & 6 & 40,00 & 1 & 6,66 & -- & -- \\
\hline
\end{tabular}

Fuente: Elaboración propia.

Se comprobó una vez desarrollado los ejercicios que los indicadores se comportaron de forma ascendente, partiendo del contrataque transición ofensiva destacando el interés mostrados por los jugadores las deficiencias principales estuvieron en la superioridad numérica y la toma de decisiones en la transición, aspecto que se debe seguir trabajando.

\section{Proceso de ejecución.}

La planificación de los ejercicios estuvo basada en los principios de la metodología periodización táctica como la supradimención táctica, incentivando la supeditación de los demás renglones del entrenamiento, al entrenar jugando y al jugar aprendiendo aspectos estos que consideramos muy importante ya que nos ayudan con la implementación y comprensión 
de la metodología, la cual nos posibilite la evolución Biopsicosocial de nuestros atletas, logrando mayor integralidad en todos los componentes del juego.

Pudimos constatar en las categorías ocupación de carriles y la continuidad de juego se comportaron de forma similares en cuanto a la aceptación en el en la ejecución de los ejercicios propuesto, los atletas se adaptaron de forma paulatina, existiendo mejor comprensión en el momento de la ocupación de los carriles, con buena reacción de respuesta en la anticipación a la defensa.

El comportamiento de las categorías superioridad numérica y toma de decisiones, fuero de interés para nuestros atletas, aunque en menor grado que en las otras dos categorías, significando que son esto dos aspectos de juego los que mayor relación guardan entre sí, y que por su comprensión y ejecución resultan las más difíciles de poder lograr en nuestro modelo de juego.

En la aplicación de la metodología existieron algunas dificultades derivadas fundamentalmente de la novedad de la misma, no obstante, de tener un buen porciento en la ocupación de carriles, no se tradujo así en la superioridad numérica, el no mantener los espacios ganados, separación entre jugadores y ritmo de juego, influyendo en la toma de decisiones con decisiones apuradas en otros casos muy demoradas creando en algunos momentos confusión, generándose menor interés en estas dos categorías.

Referenciaría comparativa del resultado antes y después de la aplicación de la metodología según nuestras categorías.

Tabla 2. Progreso y logros de la metodología

\section{(S): SIEMPRE (CS): CASI SIEMPRE (PV): POCAS VESES (N): NUNCA}

\begin{tabular}{|c|c|c|c|c|c|c|c|c|c|c|c|c|c|c|c|c|}
\hline \multirow[b]{2}{*}{ Categorías } & \multicolumn{8}{|c|}{ Primera medición } & \multicolumn{8}{|c|}{ Segunda medición } \\
\hline & $\mathbf{S}$ & $\%$ & $\begin{array}{c}\text { C } \\
\dot{S}\end{array}$ & $\%$ & $\begin{array}{l}\mathbf{P} \\
\mathbf{V}\end{array}$ & $\%$ & $\mathbf{N}$ & $\%$ & $\mathbf{S}$ & $\%$ & $\begin{array}{l}\text { C. } \\
\text { S }\end{array}$ & $\%$ & $\begin{array}{l}\mathbf{P} \\
\mathbf{V}\end{array}$ & $\%$ & $\mathbf{N}$ & $\%$ \\
\hline Ocupación de carriles & 2 & 13.3 & 2 & 13.3 & 8 & 53.3 & 3 & 20.0 & 7 & 46.6 & 7 & 46.6 & 1 & 6.6 & - & \\
\hline $\begin{array}{l}\text { Superioridad } \\
\text { numérica }\end{array}$ & 1 & 6.6 & - & & 6 & 40.0 & 8 & 53.3 & 9 & 60.0 & 3 & 20.0 & 2 & 13.3 & 1 & 6.6 \\
\hline $\begin{array}{l}\text { Toma de decisiones } \\
\text { en la transición }\end{array}$ & - & & - & & 9 & 60.0 & 6 & 40.0 & 9 & 60.0 & 4 & 26.6 & 1 & 6.6 & 1 & 6.6 \\
\hline Continuidad de juego & - & & 4 & 26.6 & 6 & 40.0 & 5 & 33.3 & 9 & 60.0 & 5 & 33.3 & 1 & 6.6 & - & \\
\hline
\end{tabular}

Fuente: Elaboración propia.

Propuesta de un esquema de ejercicios que nos permite tener una guía de trabajo con base a la periodización táctica 


\section{Ocupación de carriles}

Dividimos en tres paneles la cancha, por el carril derecho corre el 2, por el central corren 1,4 y 5 y por el izquierdo siempre se desplaza el 3 esta es la ocupación asignada a cada jugador orientando que deben llegar lo más rápido posible a la cancha contraria, los jugadores 2 y 3 su

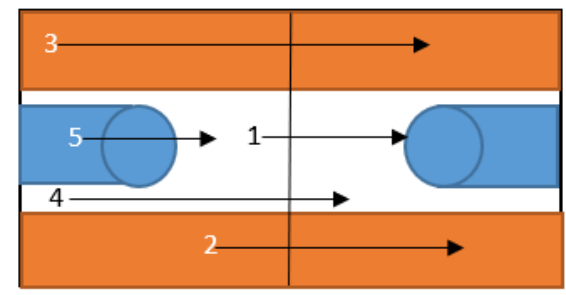
recorrido debe ser lo más próximo a las línea laterales.

de igual manera se le orienta a los jugadores que deben conocer las funciones de cada posición para poder intercambiar en un momento dado su designación y ser más versátiles, tanto de ida como de regreso mantener los carriles, por el carril derecho corre el 2, por el central corren 1,4 y 5 y por el izquierdo siempre se desplaza el 3 esta es la ocupación asignada a cada jugador orientando que deben llegar lo más rápido posible a la cancha contraria, los jugadores 2 y 3 su recorrido debe ser lo más próximo a las línea laterales.

\section{Superioridad numérica}

Vemos en la disposición inicial del ejercicio, que los jugadores 1, y 2 iniciarán el ejercicio atacando al número 3 , primera situación de ventaja numérica $2 \times 1$.

una vez finalizada la acción, los tres jugadores se dispondrán a atacar en el aro contrario, ante los defensores 9 , y 7 . es la siguiente situación de superioridad, $3 \times 2$. en todas las acciones si éstas terminan en canasta, obligaremos a sacar de fondo a

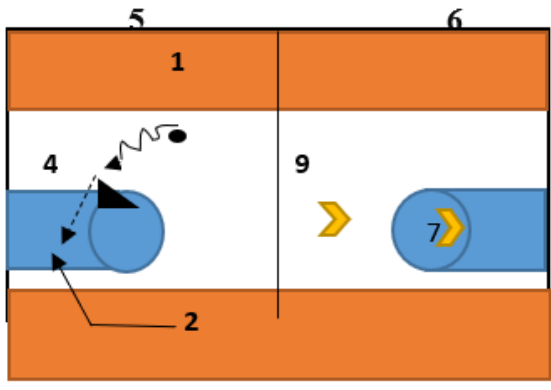

4
8 los atacantes, como lo haríamos en un partido. lo vemos en el siguiente diagrama. 
una vez finalizada la acción de 4x3, los atacantes (números 6,7,8, y 9) se convierten en defensores, y los que eran defensores (números 1,2, y 3), se convierten, junto a 8, y 6 , que están fuera, en los nuevos atacantes, finalizando el ejercicio, con la última situación de superioridad, $5 \times 4$. podemos observarlo en el siguiente diagrama:

\section{Toma de decisiones en la transición}

inicia con rebote defensivo del jugador \# 3 contra el tablero , evade la defensa le pasa a 2 que se desmarca a la prolongación del tiro libre botea buscando en centro mientras los jugadores 1 y 3 corren por los carriles externos, mientras 4, y 3, están esperando, a los atacantes en el aro contrario el jugador 2 tendrá 2 opciones pasarle a 1 o pasarle a 3 . ya tenemos montada nuestra primera situación de superioridad, en este caso $3 \times 2$. lo vemos en el diagrama:

inicia con rebote defensivo del jugador \# 3 contra el tablero, evade la defensa del jugador 6 le pasa a 2 que se desmarca a la prolongación del tiro libre recibe el pase botea buscando en centro mientras los jugadores 3,4 corren por los carriles externos, mientras los defensas, están esperando, a los nuevos atacantes en el aro contrario el jugador 2 tendrá 3 opciones pasarle a 1 esperar la pantalla de 4 a 3 para darle un pase cerca del aro o pasarle a 1 y realizar una toma y dame. ya tenemos montada nuestra tercera situación de inicia con rebote defensivo del jugador \# 3 contra el tablero, evade la defensa
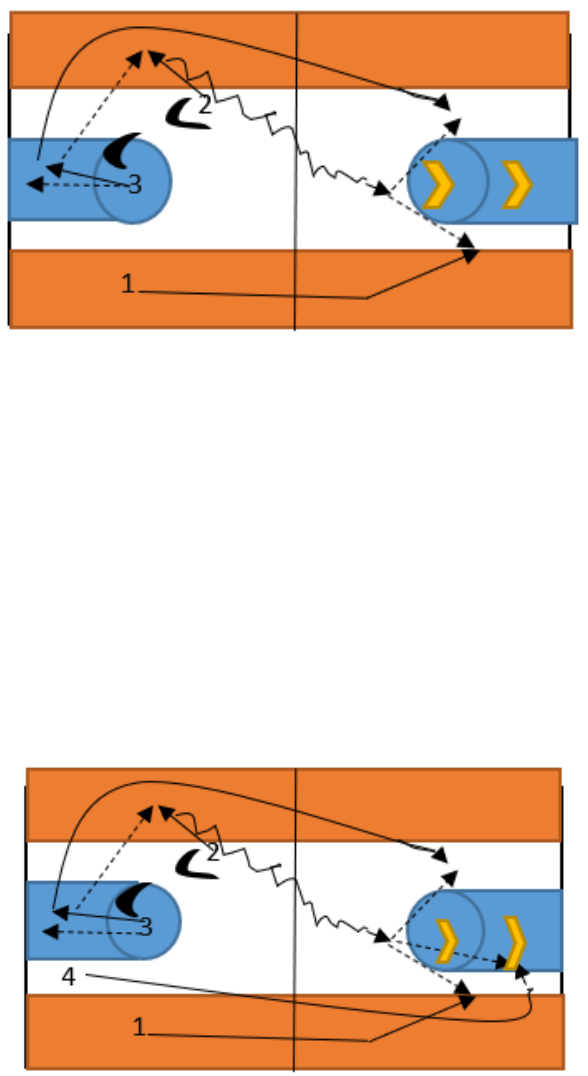
del jugador 6 le pasa a 2 que se desmarca a la prolongación del tiro libre botea buscando en centro mientras los jugadores 3,4 corren por los carriles externos, mientras 8, y 9, están esperando, a los nuevos atacantes en el aro contrario el jugador 2 tendrá 3 opciones pasarle a 1 esperar la pantalla

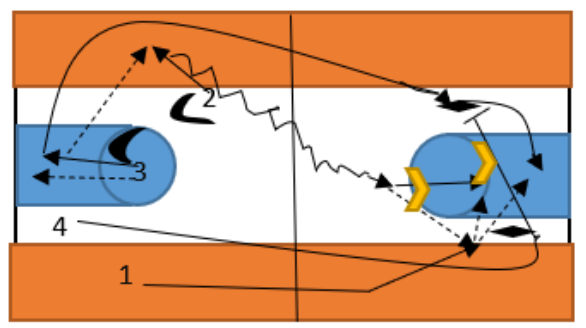
de 4 a 3 para darle un pase cerca del aro o pasarle a 1 y realizar una toma $\mathrm{y}$ dame. ya tenemos montada nuestra tercera situación de superioridad, en este caso $4 \times 2$. lo vemos en el diagrama:

\section{Continuidad de juego}

$5 \times 5 \times 5$ toda cancha

tres equipos de cinco jugadores.

se juega en toda la pista.

un equipo a media pista con balón (equipo atacante).

los otros dos equipos (defensores) colocados cada uno de ellos en diferente media pista.

jugamos situaciones de $5 \times 5 \times 5$, o sea, situaciones donde cada equipo defiende hasta media pista.

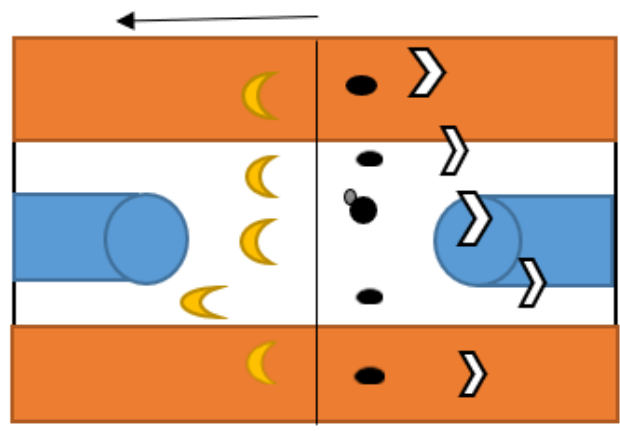

El equipo que anota sigue atacando hacía el aro contrario. si el equipo que defiende recupera el balón, sale atacando hacia el campo contrario.

Fuente: Elaboración propia.

\section{Conclusiones.}

- La principal dificulta al entrenar esta metodología esta dado, por el poco conocimiento sobre esta, los atletas a pesar de mostrar interés por lo novedoso su adaptación su comprensión es un poco más lenta que otras metodologías.

- La aplicación de la metodología nos ofrece la oportunidad de poder contar con una herramienta eficaz, que permite a nuestros atletas conocer y entrenar nuestro modelo, ya que la practica está regida por la dimensión táctica que asume como guía todos los demás componentes del juego, rectorando todo el proceso de entrenamiento. 
- Nos permite entrenar en situaciones de juego real facilitando una mejora en la toma de decisiones al estar los atletas sometidos a situaciones complejas que solo el juego constante nos permite modelar en el día a día de los entrenamientos.

- Principales contribuciones en la aplicación de la propuesta metodología.

- Mejoras en el rendimiento de las capacidades físicas, resistencia, velocidad y fuerza en relación con el trabajo en el juego.

- Mejora en la coordinación en los ritmos y tiempos del juego de forma individual y colectiva.

- Aumento de la concentración en de nuestros atletas en los juegos.

- Mejora en las interrelaciones personales de los atletas, al poder explotar sus habilidades individuales en función del equipo.

\section{Referencias bibliográficas.}

Alzola Tamayo, A., Ortiz Fernández, D., Vaca, M. E., \& Alzola Tamayo, S. (2019). Determinación estructural de la periodización táctica en el baloncesto femenino de la Espicha sede Morona Santiago Ecuador. Ciencia Digital, 3(3.1), 390-408. https://doi.org/10.33262/cienciadigital.v3i3.1.710.

Alffonso J. García F. (2018). Análisis del bloqueo directo en transición ofensiva en la Americup 2017) Tesis en opción al Título en Licenciatura en Educación Física, Recreación y Deporte. Instituto Universitario asociación cristiana de jóvenes licenciatura en Educación Física, Recreación y Deporte. Montevideo Uruguay

Castro A A. (2019). Propuesta metodológica para entrenamiento de tareas de finalización en el fútbol femenino de Holguín. Tesis en opción al título de master en Pedagogía del Deporte y la Cultura Física Universidad de Holguín. Cuba.

Davids, K., Güllich, A., Shuttleworth., R., \& Araújo, D. (2017). Understanding Environmental and Task Constraints on Talent Development, En J. Baker, S. Cobley, J. Schorer \& N. Wattie (Eds.), Routledge Handbook of Talent Identification and Development in Sport. Abingdon: Routledge.

Freyre, V F. (2018). El entrenamiento de la fuerza explosiva en el Baloncesto. Tesis en opción al grado científico de Doctor en Ciencias de la Cultura Física. Universidad de Holguín. La Habana. Cuba.

Gutiérrez, D., Fisette, J., García-López, L. M., \& Contreras, O. (2014). Assessment of Secondary School Students' Game Performance Related to Tactical Contexts. Journal of Human Kinetics, 42, 223-234.

Klimenko, O. Ayala, D Munera A y Rave, S (2020). Funciones ejecutivas, inteligencia contextual percibida y dimensión subjetiva en la toma de decisiones en deportistas con mayor y menor desempeño en las acciones ofensivas de $1 \mathrm{v} / \mathrm{s} 1$ en el equipo de Baloncesto masculino de liga juvenil. Artículo de acceso abierto publicado por: 
Revista Digital: Actividad Física y Deporte, bajo una licencia Creative Commons CC BY-NC 4.0.

Loaiza, P. G. (2017). Periodización táctica: planificación de una temporada en baloncesto de élite. Tesis en opción al Grado en Ciencias de la Actividad Física y el Deporte. Universidad de Cádiz. España.

Leica López L La transición y su incidencia en los sistemas de juego de los deportistas de baloncesto. Tesis en opción al grado científico de Maestría en Cultura Física y entrenamiento deportivo. Universidad Técnica de Ambato Ecuador.

Praxedes P. A. (2018). El diseño de tareas para el aprendizaje de las habilidades en el Fútbol de iniciación. una aplicación desde las perspectivas Cognitiva y Ecológica. Tesis en opción al grado científico de Doctor en Ciencias de la Cultura Física. Universidad de Extremadura. España.

Serra O J. (2013). Conocimiento táctico y rendimiento de juego en fútbol en niños de 8 a 12 años." Tesis en opción al título de Doctor en Ciencias de la Actividad Física y del Deporte. Universidad Católica de San Antonio. Murcia. España.

Suárez Cadenas. (2017). La toma de decisiones en baloncesto. Una propuesta de árboles decisionales para la enseñanza del bloqueo directo. ACCIÓN PSICOLÓGICA, junio 2017, vol. 14, $\mathrm{n}^{\circ}$. 1, 43-56. ISSN: 1578-908X http://dx.doi.org/10.5944/ap.14.1.19259

Suárez Cadenas, (2016) Toma de decisiones y selección de tiro en Baloncesto: herramientas para la evaluación y el entrenamiento. Tesis en opción al grado científico de Doctor en Ciencias Universidad de Granada España. URI: http://hdl.handle.net/10481/46449

Tamarit, X. (2007). ¿Qué es la "Periodización Táctica? Vivenciar el juego para condicionar el Juego. Pontevedra: Ed. Mcsports.

Valero, I. A. (2016). Control y evaluación de la táctica ofensiva de los jugadores base de la categoría 13-15 años del deporte Baloncesto. Tesis en opción al grado científico de Doctor en Ciencias de la Cultura Física. Universidad Central Martha Abreu. Villa Clara Cuba.

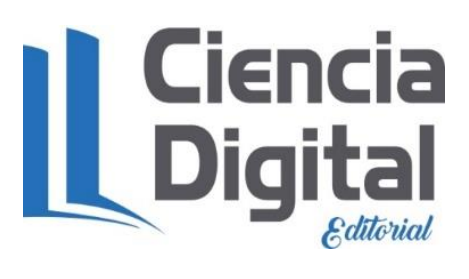


PARA CITAR EL ARTÍCULO INDEXADO.

Ortiz Fernández, D., Alzola Tamayo, A., Sánchez Anilema, J. A., \& Hechavarria Jeorge, O. (2020). Periodización táctica y la toma de decisiones en la transición ofensiva en el baloncesto femenino de la ESPOCH sede Morona Santiago Ecuador . Ciencia Digital, 4(3), 286-303. https://doi.org/10.33262/cienciadigital.v4i3.1343

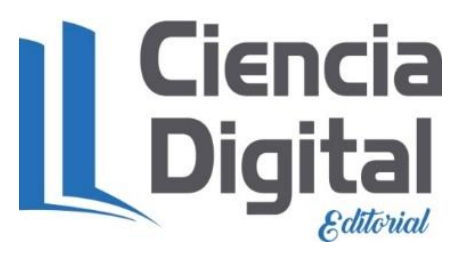

El artículo que se publica es de exclusiva responsabilidad de los autores y no necesariamente reflejan el pensamiento de la Revista Ciencia Digital.

El artículo queda en propiedad de la revista y, por tanto, su publicación parcial y/o total en otro medio tiene que ser autorizado por el director de la Revista Ciencia Digital.
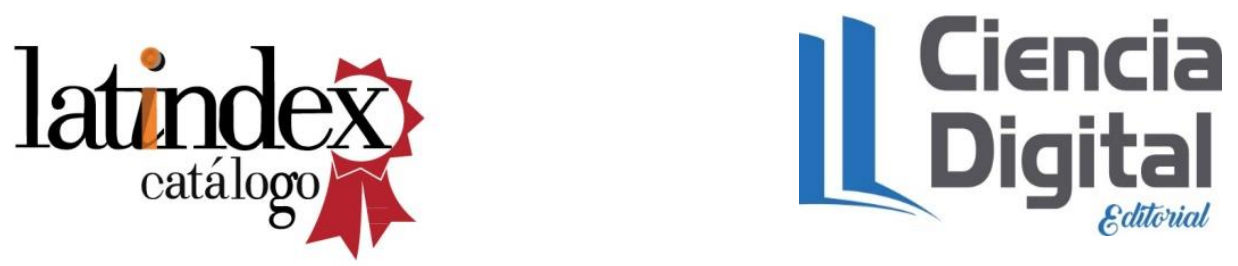